\title{
ON PREDATOR-PREY DISPERSAL, REPULSIVE DISPERSAL, AND THE PRESENCE OF SHOCK WAVES*
}

\author{
BY \\ MICHIEL BERTSCH (University of Leiden, The Netherlands) \\ AND \\ MORTON E. GURTIN (Carnegie-Mellon University)
}

1. Introduction. In this paper we study a system of partial differential equations introduced by MacCamy [6] as a model for predator-prey interactions. This system, in one space dimension, has the form

$$
\begin{aligned}
& u_{t}=\left(u v_{x}\right)_{x}, \\
& v_{t}=-\left(v u_{x}\right)_{x},
\end{aligned}
$$

and is based on the assumption that dispersal is completely controlled by the predator-prey interaction and occurs on a time scale sufficiently fast that births and deaths are negligible. Here $u(x, t)$ and $v(x, t)$ are the spatial densities of the species (and as such will always be constrained to satisfy $u, v \geqslant 0), x \in \mathbb{R}$ denotes spatial position, $t>0$ is the time, and subscripts indicate partial differentiation with respect to the corresponding variable.

If we write the right-hand side of (1.1) as a matrix $M(u, v)$ times the column vector $\left(u_{x x}, v_{x x}\right)$ plus a term involving derivatives of lower order, then

$$
M(u, v) \text { has eigenvalues } \pm i \sqrt{u v},
$$

and the system cannot easily be classified, as it is of no specific type. Because of this we expect (1.1) to exhibit nontypical behavior, and it is our purpose here to verify this expectation by deducing several explicit solutions with interesting properties. In particular, we deduce solutions which contain shocks (jumps in $u$ and $v$ ) and solutions which "blow up" to delta functions in finite time. Moreover, in one solution the shocks develop from continuous initial data. 
We also present a solution for the system

$$
\begin{aligned}
& u_{t}=\left(u v_{x}\right)_{x}, \\
& v_{t}=\left(v u_{x}\right)_{x},
\end{aligned}
$$

a system which arises as a limiting case when studying populations that disperse to avoid crowding (see [3]). Here the species are repulsive, as each disperses as a response to population pressure of the other. In this instance

$$
M(u, v) \text { has eigenvalues } \pm \sqrt{u v}
$$

and, as before, we find a solution with shocks. The behavior of this solution is quite interesting (see [3], Sec. 4, Case (iii)): the species are initially mixed but segregate in finite time.

Whether the initial-value problems for the systems (1.1) and (1.3) are well-posed is an open question well beyond the scope of this paper. For (1.3) well-posedness cannot be expected, since here $M(u, v)$ has a negative eigenvalue. Further, the analogous linear system

$$
\begin{aligned}
& u_{t}=v_{x x}, \\
& v_{t}=u_{x x}
\end{aligned}
$$

is clearly ill-posed, since $u-v$ satisfies the backward heat equation.

For completeness we give a careful derivation of the systems (1.1) and (1.3) together with some related results concerning shock waves. In an appendix we give the precise sense in which $u$ and $v$ solve the underlying initial-value problems.

2. Basic equations. As with most continuum theories, our theory makes use of conservation laws of the form

$$
\frac{d}{d t} \int_{a}^{b} f(x, t) d x=h(b, t)-h(a, t),
$$

where, for a particular species, $f(x, t)$ represents the spatial density and $h(x, t)$ the population flux. More precisely, $f(x, t)$ is the number of individuals, per unit length, at $x$, while $h(x, t)$ is the number of individuals that cross $x$ (from right to left) per unit time. When the dispersal velocity is known, $h$ is given by the formula

$$
h=-(\text { density)(dispersal velocity). }
$$

To avoid repetition, we say that $f$ and $h$ balance if (2.1) holds for all $a, b \in \mathbb{R}$ and $t>0$. When this is the case, $f$ and $h$ satisfy the local conservation law

$$
f_{t}=h_{x}
$$

wherever smooth.

We here consider the dispersal of two biological species in $\mathbb{R}$. The species are assumed to have spatial densities $u(x, t)$ and $v(x, t)$ and dispersal velocities $q(x, t)$ and $g(x, t)$, respectively. We assume further that the species disperse sufficiently fast that population changes due to births and deaths are negligible. Then balance of population requires that $u$ and - uq balance, and that $v$ and -vg balance. 
a. Predator-prey. Let the predator have density $v$, the prey density $u$. We model the predator-prey interaction by means of the constitutive equations

$$
\begin{aligned}
& q=-v_{x}, \\
& g=u_{x},
\end{aligned}
$$

so that the prey disperse in the direction of decreasing predator population, the predators in the direction of increasing prey population. (There is no loss of generality in not putting strictly positive constants before $v_{x}$ and $u_{x}$, since the form of the conservation law allows us to remove such constants by rescaling $u$ and $v$.) In the presence of sufficient smoothness, the conservation law and constitutive equations together yield the following system of partial differential equations:

$$
\begin{aligned}
& u_{t}=\left(u v_{x}\right)_{x}, \\
& v_{t}=-\left(v u_{x}\right)_{x} .
\end{aligned}
$$

An interesting feature of the system (2.3) is invariance under the transformation

$$
u \rightarrow v, \quad v \rightarrow u, \quad t \rightarrow T-t .
$$

b. Repulsive species. Consider two species, each of which disperses away from the other. ${ }^{1}$ A possible model for such behavior is based on the assumption that the velocity of $u$ be proportional to $v_{x}$ and point in the direction of decreasing $v$, and similarly for the velocity of $v$. More precisely, we take as our constitutive assumption

$$
\begin{aligned}
& q=-v_{x}, \\
& g=-u_{x},
\end{aligned}
$$

which leads to the partial differential equations

$$
\begin{aligned}
& u_{t}=\left(u v_{x}\right)_{x}, \\
& v_{t}=\left(v u_{x}\right)_{x} .
\end{aligned}
$$

3. Relations across shocks. To study both of the foregoing systems simultaneously, we rewrite the constitutive equations as

$$
\begin{aligned}
& q=-v_{x}, \\
& g=-k u_{x}
\end{aligned}
$$

with

$$
k=-1 \text { predator-prey, } k=1 \text { repulsive species. }
$$

The underlying partial differential equations are then

$$
\begin{aligned}
& u_{t}=\left(u v_{x}\right)_{x}, \\
& v_{t}=k\left(v u_{x}\right)_{x} .
\end{aligned}
$$

\footnotetext{
${ }^{1}$ See $[3,5]$ for a model in which dispersal is driven by total population.
} 
We will always use the term solution ${ }^{2}$ to signify that $u$ and $-u q$ balance, as do $v$ and $-v g$, with $q$ and $g$ given by (3.1). The solutions we will discuss will contain shock waves, that is, moving fronts $x=X(t)$ across which $u$ or $v$ suffers jump discontinuities. As is well known, to ensure that the conservation law (2.1) is satisfied we must adjoin - to the system (3.2), which is required to hold away from all shocks - the jump condition(s)

$$
\dot{X}[f]=-[h]
$$

across each shock (e.g., see [4], Sec. 73). Here and in what follows we use the standard notation

$$
[f]=f^{+}-f^{-}, \quad f^{ \pm}(t)=f(X(t) \pm 0, t) .
$$

To apply the theory of conservation laws, $f$ and $h$ in (2.1) must be functions. Consider the conservation law for $u$ in which $f=u$ and $h=u v_{x}$. If $v$ suffers a jump discontinuity, then $v_{x}$ is a distribution; this will not be a problem, however, provided $u$ is continuous with value zero at $x=X(t)$, for then $u v_{x}$ behaves as a function. A similar argument applies to the conservation law for $v$. We therefore restrict our attention to the following two types of shocks:

(i) $u$-shock for which

$$
[u] \neq 0, \quad v^{+}=v^{-}=0
$$

(ii) $v$-shock for which

$$
[v] \neq 0, \quad u^{+}=u^{-}=0 .
$$

When discussing the predator-prey system we will use the term prey shock rather than $u$-shock, predator shock rather than $v$-shock. Trivially, the jump condition (3.3) yields two relations:

$$
\begin{aligned}
& \dot{X}[u]=-\left[u v_{x}\right], \\
& \dot{X}[v]=-k\left[v u_{x}\right] .
\end{aligned}
$$

However, in each of the cases (i) and (ii) we need only consider one of (3.6), since

$(3.6)_{1}$ is satisfied automatically

for a $v$-shock, $(3.6)_{2}$ for a $u$-shock.

Several results hold for shocks that move into, or out of, unpopulated regions. Thus assume, for the remainder of the section, that $u(x, t)$ and $v(x, t)$ vanish for $x>X(t)$ or for $x<X(t)$, at least for $x$ sufficiently close to $X(t)$. We will say that the region to the right or left, respectively, is unpopulated to signify these two possibilities. In view of (3.4), (3.5), and (3.7), the jump conditions (3.6) are together equivalent to the single relation

$$
\dot{X}=-k u_{x}^{ \pm}
$$

for a $v$-shock, and to the single relation

$$
\dot{X}=-v_{x}^{ \pm}
$$

\footnotetext{
${ }^{2}$ For $u$ and $v$ smooth except for shocks, this definition is equivalent to requiring that $u$ and $v$ satisfy (3.2) in the sense of distributions (see the Appendix).
} 
for a $u$-shock, where in (3.8) and (3.9) the plus or minus sign is chosen according as the region to the left or right is unpopulated.

Consider now a $v$-shock with the region to the left unpopulated. Since $u$ is continuous across $x=X(t)$, while $u_{x}$ and $u_{t}$ suffer at most jump discontinuities, we have the classical compatibility condition (e.g., see [4], p. 250)

$$
\left[u_{t}\right]=-\dot{X}\left[u_{x}\right] \text {. }
$$

Further, by (3.2) 1 and (3.5),

$$
u_{t}^{+}=u_{x}^{+} v_{x}^{+} .
$$

Also, for $\dot{X} \neq 0$ we may use (3.8) to conclude that $u_{x}^{+} \neq 0$; hence the last two relations imply that $\dot{X}=-v_{x}^{+}$. Similar relations apply to the other cases of interest, and therefore, appealing to (3.8) and (3.9), we are led to the following

Proposition. Assume that $\dot{X} \neq 0$. Then

$$
\dot{X}=-k u_{x}^{ \pm}=-v_{x}^{ \pm}
$$

with the plus or minus signs chosen according as the region to the left or right is unpopulated.

In view of the constitutive equation (3.1), the relations (3.10) have the following interpretation: the dispersal velocities of the two species at the shock and the velocity of the shock coincide. (Such shocks are therefore contact discontinuities.)

\section{Solutions of the predator-prey system}

a. Similarity solutions. Following $[2,5,7]$, we seek similarity solutions of the form

$$
\begin{aligned}
u(x, t) & =t^{-1 / 3} U(\xi), \\
v(x, t) & =t^{-1 / 3} V(\xi), \\
\xi & =t^{-1 / 3} x .
\end{aligned}
$$

Substituting these relations into (2.3) leads to the equations

$$
(\xi U)^{\prime}=-3\left(U V^{\prime}\right)^{\prime}, \quad(\xi V)^{\prime}=3\left(V U^{\prime}\right)^{\prime} .
$$

Here we do not seek the most general similarity solution, but rather a simple solution with interesting properties; we therefore satisfy (4.2) by taking

$$
\begin{aligned}
& U(\xi)=C_{1}+\frac{\xi^{2}}{6}, \\
& V(\xi)=C_{2}-\frac{\xi^{2}}{6} .
\end{aligned}
$$

These functions cannot yield solutions for all $\xi$, as $V(\xi)$ is negative for $|\xi|$ large. We can, however, use pieces of these functions to generate solutions on all space. We consider two cases.

Case 1. $C_{1}>0, C_{2}>0$. Let

$$
C_{1}=\frac{P^{2}}{6}, \quad C_{2}=\frac{Q^{2}}{6}
$$


with $P, \dot{Q}>0$. Then $U(\xi)>0$ always, so we construct our solution using two prey shocks:

$$
\begin{gathered}
U(\xi)=\frac{1}{6}\left(P^{2}+\xi^{2}\right), \quad V(\xi)=\frac{1}{6}\left(Q^{2}-\xi^{2}\right) \text { for }-Q<\xi<Q, \\
U(\xi)=V(\xi)=0 \text { otherwise. }
\end{gathered}
$$

For this choice of $U$ and $V$ there are shocks at $x= \pm Q t^{1 / 3}$, across which (3.9) is satisfied. Thus the corresponding $u$ and $v$ form a solution on $\mathbb{R}$ for all $t>0$ (see Fig. 1).

Case 2. $C_{1} \leqslant 0, C_{2}>0$. Let

$$
C_{1}=-\frac{P^{2}}{6}, \quad C_{2}=\frac{Q^{2}}{6}
$$

For $Q \leqslant P$ the sets $\{U>0\}$ and $\{V>0\}$ are disjoint, so we need consider only $P<Q$. Let

$$
\begin{gathered}
U(\xi)=\frac{1}{6}\left(\xi^{2}-P^{2}\right), \quad V(\xi)=\frac{1}{6}\left(Q^{2}-\xi^{2}\right) \quad \text { for } P<\xi<Q, \\
U(\xi)=V(\xi)=0 \quad \text { otherwise. }
\end{gathered}
$$

Here $x=Q t^{1 / 3}$ is a prey shock, $x=P t^{1 / 3}$ a predator shock, with (3.9) satisfied across the former, (3.8) across the latter, so we again have a solution (see Fig. 2). We also have a similar solution supported in $-Q \leqslant \xi \leqslant-P$ and a third solution obtained by adding this and (4.4).

Consider the solution $u, v$ defined by (4.1) and (4.3). In view of the invariance (2.4), given any $T>0$,

$$
\begin{aligned}
& \hat{u}(x, t)=v(x, T-t), \\
& \hat{v}(x, t)=u(x, T-t)
\end{aligned}
$$

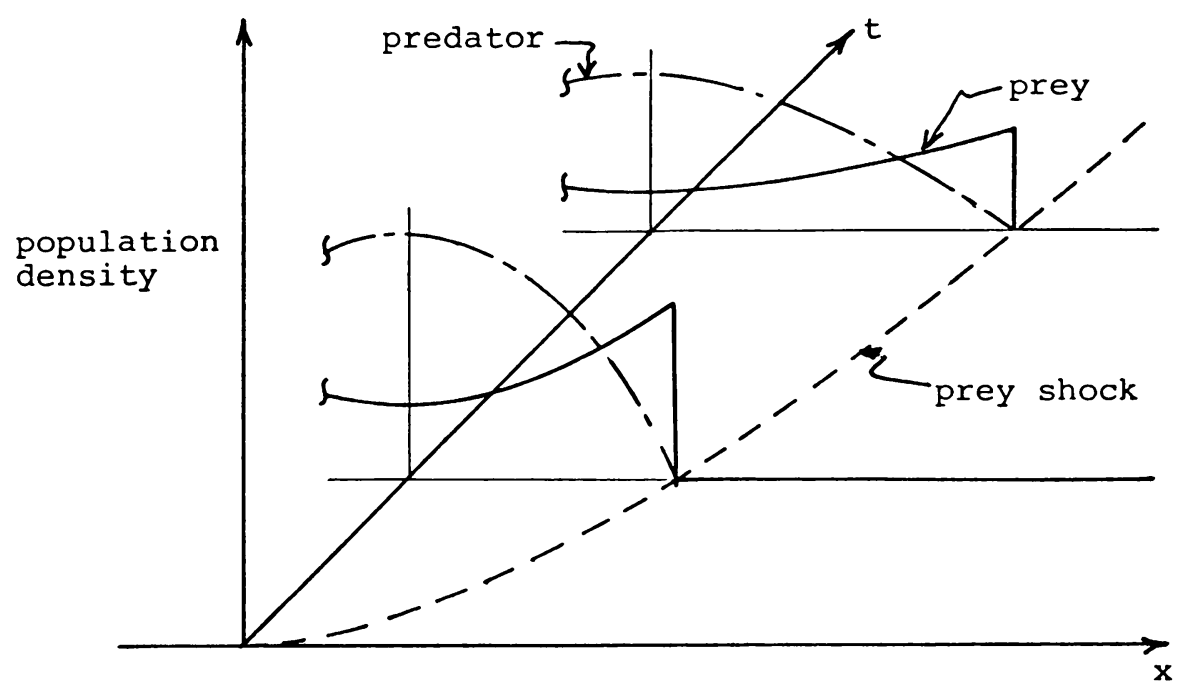

FIG. 1. Predator and prey densities for Case 1 at two fixed times as functions of $x$. The densities are not shown for $x<0$, as they are mirror images of those for $x>0$. 


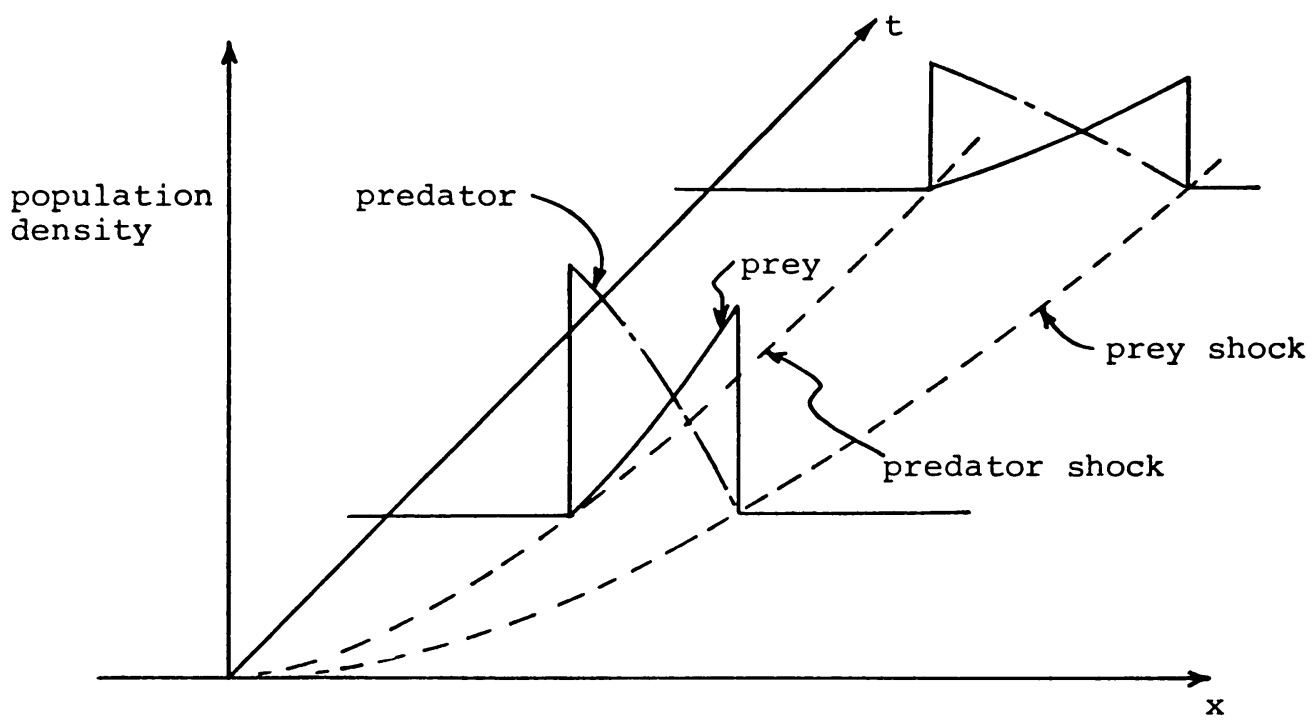

FIG. 2. Predator and prey densities for Case 2 at two fixed times as functions of $x$.

is a solution on $\mathbb{R} \times(-\infty, T)$. This new solution has an interesting property: as $t \uparrow T$,

$$
\hat{u}(x, i), \hat{v}(x, t) \rightarrow \begin{cases}\infty & \text { if } x=0 \\ 0 & \text { otherwise. }\end{cases}
$$

In fact, as $t \uparrow T, \hat{u}(\cdot, t)$ and $\hat{v}(\cdot, t)$ approach Dirac distributions (delta functions).

By similar transformations of the solutions of Case 2 we can generate other solutions which "blow up" in finite time.

b. Piecewise-affine solutions. We begin with some local solutions of the system (2.3). Here $u(x, t)$ and $v(x, t)$ are defined on an open set in $\mathbb{R} \times(0, \infty)$.

( $\alpha$ ) If $u$ is independent of time and $v \equiv 0$, or vice versa, then $u$ and $v$ solve (2.3).

$(\beta)$ When affine, $u$ and $v$ solve (2.3) if and only if

$$
u(x, t)=A(x+B t)+C, \quad v(x, t)=B(x-A t)+D,
$$

with $A, B, C$, and $D$ constants.

The result $(\alpha)$ is immediate, while $(\beta)$ follows from the fact that, for $u$ and $v$ affine, (2.3) is equivalent to

$$
u_{t}=u_{x} v_{x}=-v_{t} .
$$

Consider the initial conditions

$$
\begin{aligned}
& u(x, 0)= \begin{cases}0, & x<0 \\
a x, & x \geqslant 0\end{cases} \\
& v(x, 0)= \begin{cases}0, & x<0 \\
b x, & x \geqslant 0\end{cases}
\end{aligned}
$$


with $a, b>0$ constants. We expect the predators to move into the field of prey, leaving behind stationary prey; we therefore look for a solution of the form shown in Fig. 3. By $(\alpha)$ and $(\beta)$, Eqs. (2.3) will be satisfied in each of the three regions, and the initial conditions (4.5) will hold for $x \neq 0$ provided only that, in Region III,

$$
\begin{aligned}
& u(x, t)=a(x+b t), \\
& v(x, t)=b(x-a t) .
\end{aligned}
$$

As is clear from Fig. 3, we should allow for a prey shock $\mathscr{S}(\mathrm{I}, \mathrm{II})$ separating regions I and II and for a prey shock $\mathscr{S}$ (II, III) separating II and III. Thus $v$ should be continuous and, by (3.7), the shocks should be consistent with (3.6) ${ }_{1} . \mathscr{S}(\mathrm{I}, \mathrm{II})$ trivially has this property, since $\dot{X}=0$ and $v_{x}^{ \pm}=0$. Thus consider $\mathscr{S}$ (II, III). Since $v$ must be continuous across this shock, we must have

$$
X(t)=a t,
$$

and the jump condition (3.6) 1 yields

$$
E=0, \quad F=(a+b)^{2} / a .
$$

Thus the solution is (see Fig. 4):

Region I: $\quad u=v=0$

Region II: $\quad u(x)=\frac{(a+b)^{2}}{a} x, \quad v=0$

Region III: $\quad u(x, t)=a(x+b t), \quad v(x, t)=b(x-a t)$

$\mathscr{S}(\mathrm{II}, \mathrm{III}): \quad x=a t$.

Note that $\mathscr{S}(\mathrm{I}, \mathrm{II})$ is not a shock, as $u$ and $v$ are continuous there. Note also that the predator distribution is a traveling wave, as $v(x, t)$ is a function of $x-a t$ for all $x \in \mathbb{R}$ and $t>0$. In the next subsection we will give a solution in which the predator and prey distributions form a single traveling wave.

c. A traveling-wave solution. Let

$$
w(x)= \begin{cases}a x, & 0<x<L \\ 0, & \text { otherwise }\end{cases}
$$

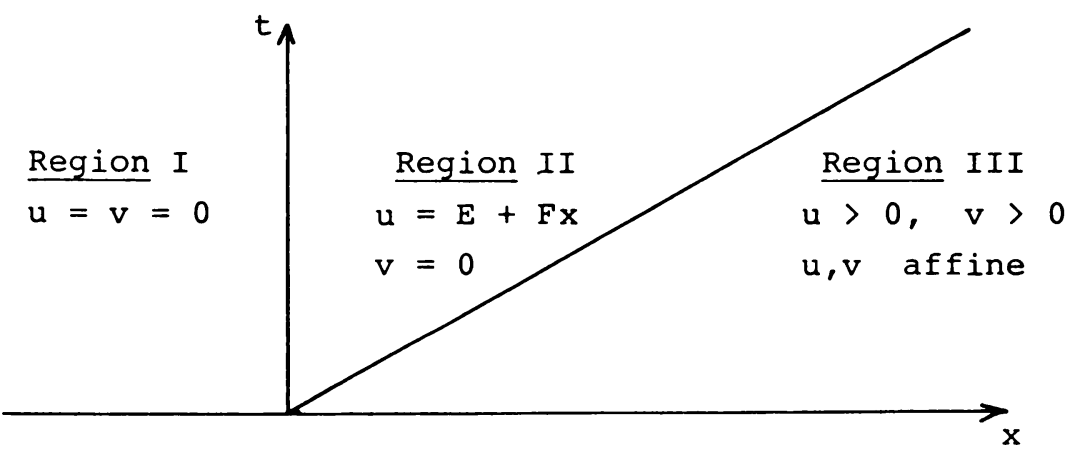

FIG. 3. Regions I-III. 
with $a, L>0$ constants. Then the functions

$$
\begin{aligned}
& u(x, t)=w(x-a t) \\
& v(x, t)=w(L-x+a t)
\end{aligned}
$$

satisfy the partial differential equations (2.3) away from the shocks

$$
x=a t, \quad x=L+a t
$$

[see ( $\beta)$ of Sec. 4b] and are consistent with the jump conditions (3.8) and (3.9) across the shocks. We therefore have a solution on $\mathbb{R}$ for all time (see Fig. 5).
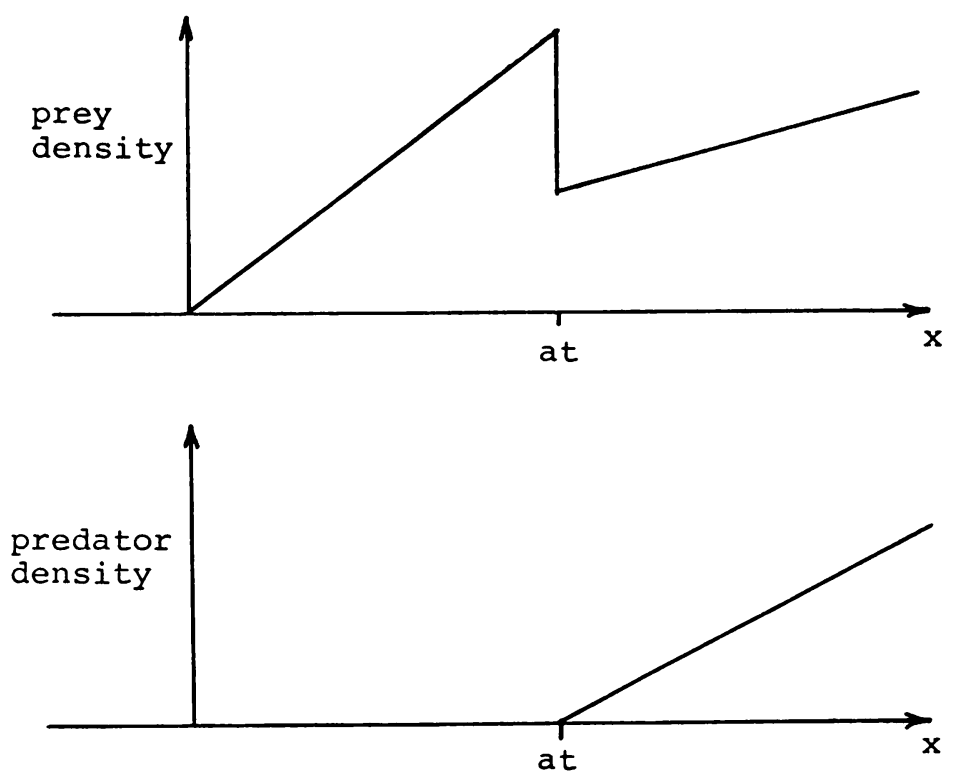

Fig. 4. Predator and prey densities as functions of $x$ for $t$ fixed.

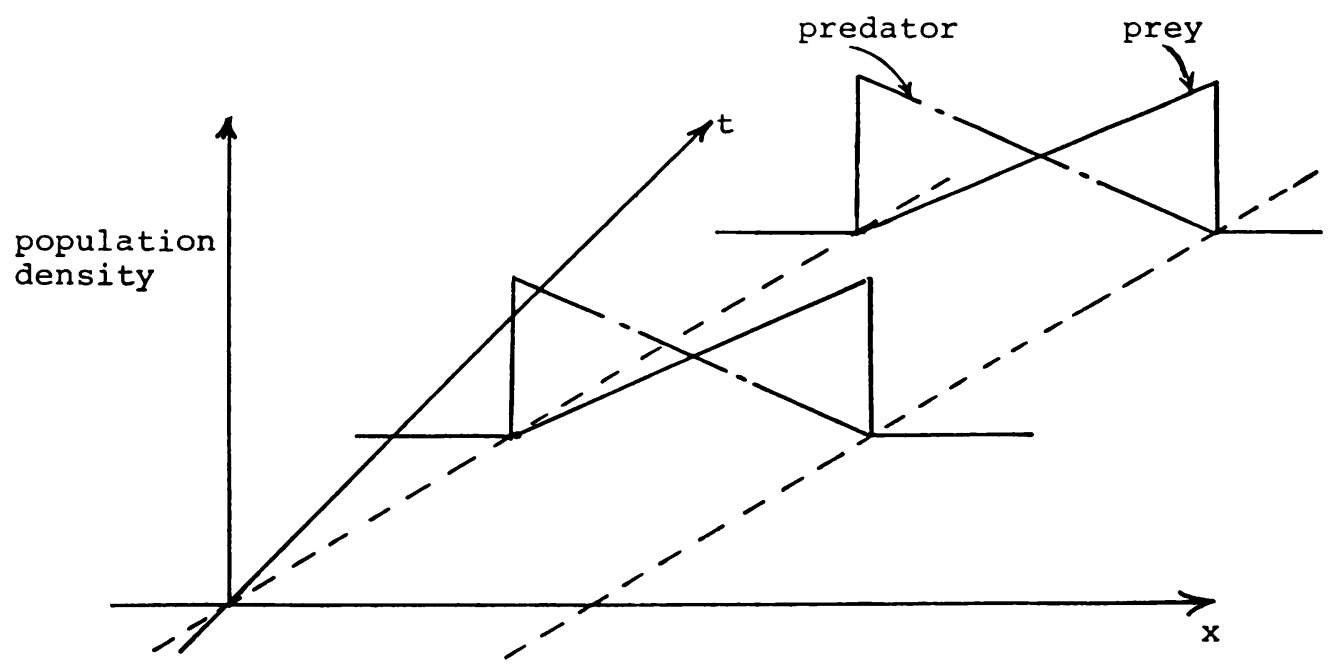

FIG. 5. Predator and prey traveling waves at two fixed times as functions of $x$. 
5. Repulsive dispersal: a solution that segregates. Here we consider the system (2.6) with piecewise-linear initial conditions

$$
\begin{aligned}
& u(x, 0)=u_{0}(x)= \begin{cases}a(L-x), & x \leqslant L \\
0, & \text { otherwise, }\end{cases} \\
& v(x, 0)=v_{0}(x)= \begin{cases}b(L+x), & x \geqslant-L \\
0, & \text { otherwise, }\end{cases}
\end{aligned}
$$

$a, b, L>0$ constants, so that initially the two species are mixed in the interval $(-L, L)$, segregated otherwise.

As in Sec. 4b we construct a piecewise-affine solution using the results $(\alpha)$ and $(\beta)$ in conjunction with the relevant jump conditions. We shall simply present the resulting solution, and to do this succintly we introduce the constants

$$
A=a+b, \quad B=a-b, \quad T=2 L / A, \quad Y=B L / A,
$$

and the functions

$$
\begin{aligned}
\bar{u}(x) & =(A / a)(B L-A x), \quad \bar{v}(x)=(A / b)(A x-B L), \\
\tilde{u}(x, t) & =a(L-x-b t), \quad \tilde{v}(x, t)=b(L+x-a t) .
\end{aligned}
$$

The solution is given in Fig. 6. There are two shocks: a $u$-shock $x=-L+a t$ and a $v$-shock $x=L-b t$; these shocks are present for times $0<t<T$ and coalesce at $t=T$ (see Fig. 7).

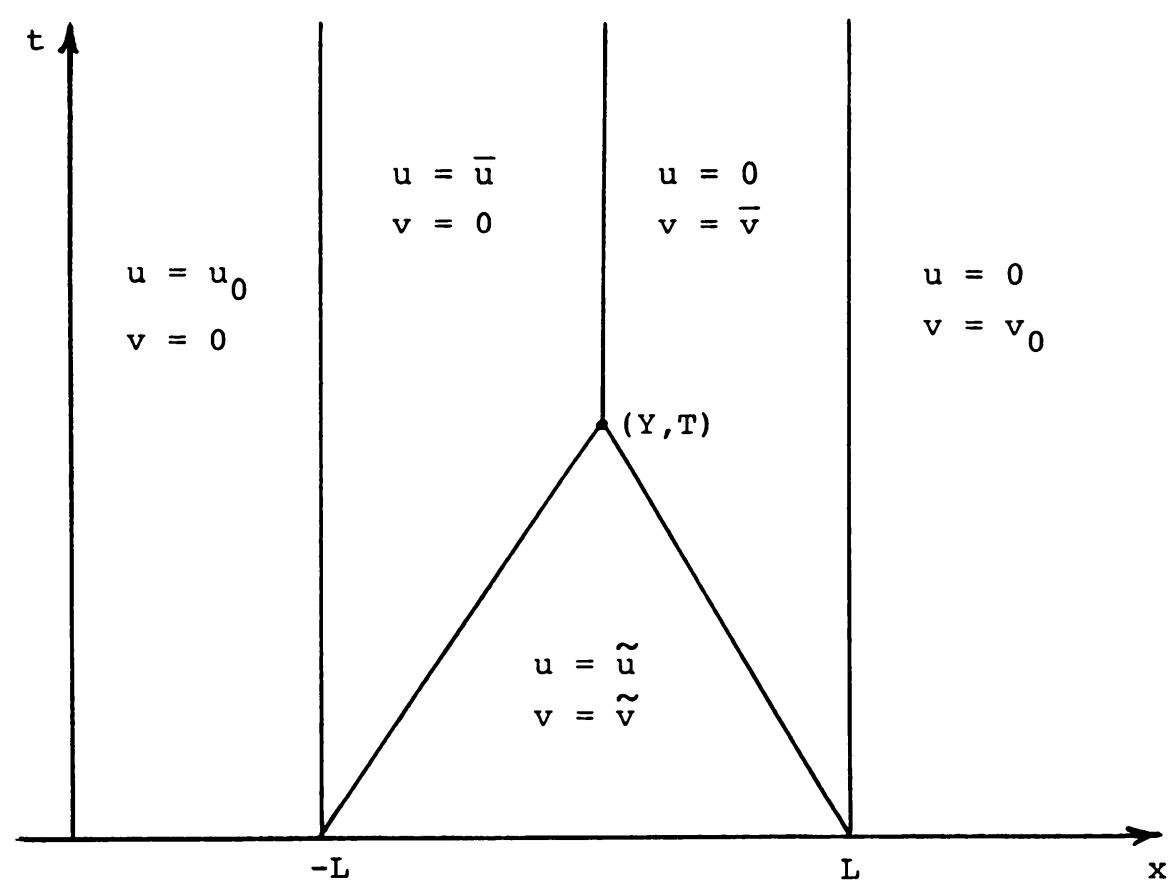

FIG. 6. The solution. 
The most interesting feature of this solution is that for times $t \geqslant T$ the two species are segregated and at equilibrium (see Fig. 7).

Note also that outside the region $(-L, L)$ the species do not disperse. In this connection, the restrictions of $u$ and $v$ to $\{(x, t):-L \leqslant x \leqslant L, t>0\}$ furnish a solution corresponding to an isolated habitat $(-L, L)$, as there is no population flux across the boundary points $x= \pm L$ :

$$
u v_{x}=v u_{x}=0 \quad \text { at } x= \pm L \text { for } t>0 .
$$
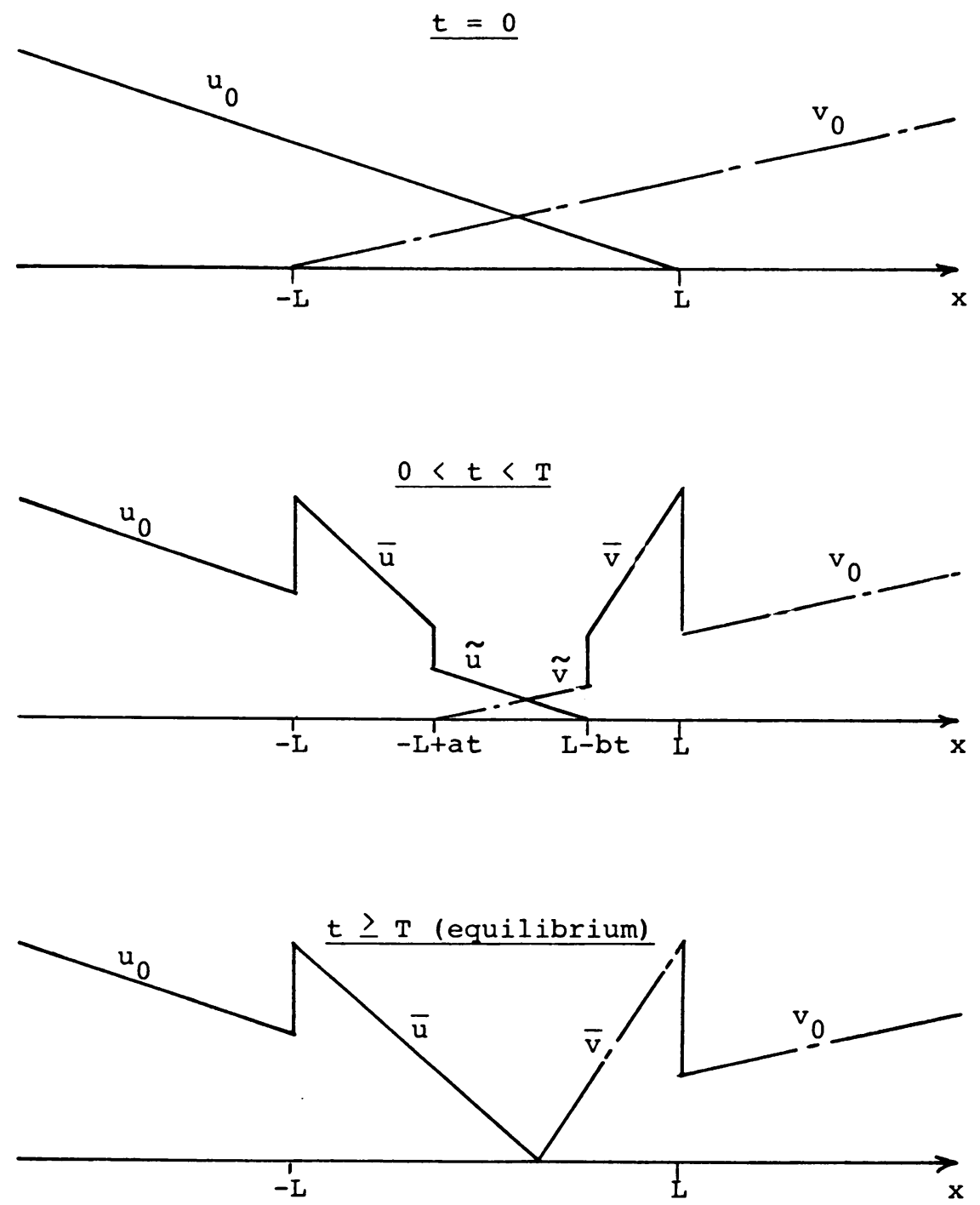

FIG. 7. $u(x, t)$ and $v(x, t)$ as functions of $x$ initially, in transition $(0<t<T)$, and at equilibrium $(t \geqslant T)$. 
Acknowledgment. This work was supported in part (M.E.G.) by the National Science Foundation.

Appendix on weak solutions. Let

$$
Q=\mathbb{R} \times(0, \infty), \quad Q_{T}=\mathbb{R} \times(0, T),
$$

and consider the initial-value problem

$$
\mathscr{P}\left\{\begin{array}{l}
u_{t}=\left(u v_{x}\right)_{x}, \quad v_{t}=k\left(v u_{x}\right)_{x} \quad \text { in } Q, \\
u(x, 0)=u_{0}(x), \quad v(x, 0)=v_{0}(x) \quad \text { for } x \in \mathbb{R},
\end{array}\right.
$$

with $^{1} u_{0}, v_{0} \in L_{\text {loc }}^{\infty}(\mathbb{R})$ or, more generally, with $u_{0}, v_{0}$ distributions.

DEFINITION. The pair $(u, v)$ is a weak solution ${ }^{2}$ of $(\mathscr{P})$ if for

$$
f=u, \quad f_{0}=u_{0}, \quad h=u v_{x}
$$

and for

$$
f=v, \quad f_{0}=v_{0}, \quad h=k v u_{x}
$$

we have ${ }^{3}$

(i) $f, h \in L_{\text {loc }}^{\infty}(Q), f(t) \in L_{\text {loc }}^{\infty}(\mathbb{R})$ for all $t>0$;

(ii) $f \geqslant 0$ a.e. in $Q$;

(iii) given any $\psi \in C^{1}(\bar{Q})$ with $\psi(t) \in C_{0}^{1}(\mathbb{R})$ for all $t \geqslant 0$, and any $T>0$,

$$
\int_{\mathbf{R}}\left[f(T) \psi(T)-f_{0} \psi(0)\right]=\int_{Q_{T}}\left[f \psi_{t}-h \psi_{x}\right]
$$

Each of the solutions presented previously is a weak solution in the above sense. The initial conditions for Sec. $4 \mathrm{c}$ are obvious. For the similarity solutions of Sec. $4 \mathrm{a}$ the initial data are

$$
u_{0}(x)=A \delta(x), \quad v_{0}(x)=B \delta(x),
$$

with $\delta$ the Dirac distribution (delta function) and

$$
A=\int_{\mathbf{R}} U(\xi) d \xi, \quad B=\int_{\mathbf{R}} V(\xi) d \xi
$$

\section{REFERENCES}

[1] R. A. Adams, Sobolev spaces, Academic Press, New York (1975)

[2] G. I. Baranblatt, On certain nonstationary motions of liquids and gases in porous media, Prikl. Mat. Mekh. 16, 57-78 (1952)

\footnotetext{
${ }^{1}$ The function spaces are as defined in [1].

${ }^{2}$ See [8], pp. 246-248, where the jump conditions are deduced from the notion of a weak solution.

${ }^{3}$ For $F(x, t)$ we write $F(t)=F(\cdot, t)$.
} 
[3] M. Bertsch, M. E. Gurtin, D. Hilhorst and L. A. Peletier, On interacting populations that disperse to avoid crowding: preservation of segregation, J. Math. Biol. 23, 1-13 (1985)

[4] M. E. Gurtin, The linear theory of elasticity, Handbuch der Physik (C. Truesdell, ed.), vol. VIa/2, Springer-Verlag, Berlin (1972)

[5] M. E. Gurtin and A. C. Pipkin, A note on interacting populations that disperse to avoid crowding, Quart. Appl. Math. 42, 87-94 (1984)

[6] R. C. MacCamy, Simple population models with diffusion, Comp. Math. Appl. 9, 341-344 (1982)

[7] R. E. Pattle, Diffusion from an instantaneous point source with concentration-dependent coefficient, Quart. J. Mech. Appl. Math. 12, 407-409 (1959)

[8] J. Smoller, Shock waves and reaction-diffusion equations, Springer-Verlag, Berlin (1983) 